\title{
Relationship between low-frequency electric-field fluctuations and ion conics around the cusp/cleft region
}

\author{
W. Miyake ${ }^{1}$, A. Matsuoka ${ }^{2}$, and T. Mukai ${ }^{2}$ \\ ${ }^{1}$ National Institute of Information and Communications Technology, 4-2-1, Nukui-kita, Koganei, Tokyo 184-8795, Japan \\ ${ }^{2}$ Institute of Space and Astronautical Science, 3-1-1, Yoshinodai, Sagamihara, 229-8510, Japan
}

Received: 22 September 2005 - Accepted: 16 January 2006 - Published: 23 March 2006

\begin{abstract}
We investigated the relationship between lowfrequency $(0.2-4.0 \mathrm{~Hz})$ electric-field fluctuations (LEFs) and ion conics around the dayside cusp/cleft region in the altitude range from 5000 to $10000 \mathrm{~km}$ from observations made by the Akebono satellite. Ion conics were generally associated with intense LEFs. We found a significant correlation between the power spectral density of LEFs at any frequency and the energy of simultaneously observed ion conics. Ion conics with a conic angle near $90 \mathrm{deg}$ and those more aligned with magnetic field lines both had an equivalent correlation with the local intensity of the LEFs. The LEFs associated with near-perpendicular ion conics were, however, generally more intense than those associated with folded conics. The difference was clearer for low-energy conics. These results are in agreement with a scenario of height-integrated heating of ions and energization of ions by electromagnetic energy supplied by local LEFs. Ions generally stay in the energization region during their upward motion along the field line, so that more folded ion conics with weak energization reach the same energy level as near-perpendicular conics with strong energization, due to the difference in integration time. The limit on residence time in the intense heating region causes the clearer difference for low-energy conics. We set up a simple model to examine the relationship between the energization rate and the evolution of ion conics along the field lines, and obtained good agreement with the observation results.
\end{abstract}

Keywords. Ionosphere (Particle acceleration) - Magnetospheric physics (Magnetopause, cusp, and boundary layers; Magnetosphere-ionosphere interactions)

\section{Introduction}

Ion conics have an angular distribution with flux maxima at a nearly constant pitch angle over an extended energy range,

Correspondence to: W. Miyake

(miyake@ nict.go.jp) i.e. tens of $\mathrm{eV}$ to $\mathrm{keV}$. The simple explanation for their generation is perpendicular energization of polar ionospheric ions at low altitudes and the subsequent upward motion of the ions with the folding of the conic angle in the geomagnetic field. Various wave-particle interaction processes have been proposed as possible mechanisms for the perpendicular energization of ions (for a review, see Andre and Yau, 1997).

Although ion conics are observed along the auroral oval (Gorney et al., 1981), dayside enhancement of their occurrence, especially around the dayside cusp/cleft region, has been reported by several authors (Gorney et al., 1981; Kondo et al., 1990; Peterson et al., 1992; Miyake et al., 1993, 1996). Dayside ion conics are hence thought to be energized by an input of energy from the dayside magnetospheric boundary region. This suggests that there are various mechanisms of transverse ion energization in different areas of the auroral oval. Broad-band, extremely low-frequency (ELF) waves are most likely responsible for the energization of dayside ion conics (Norqvist et al., 1998; Andre and Yau, 1997), though we do not know the origin of the waves, i.e. whether they are locally excited or propagate down from the magnetosphere.

The dayside cusp/cleft region is also known for the presence of intense electric-field fluctuations (e.g. Maynard et al., 1982; Block et al., 1987; Marklund et al., 1990; Matsuoka et al., 1991, 1993; Stasiewicz et al., 2000; Ivchenko and Marklund, 2001). Maynard et al. (1982) found that the region of electric-field fluctuations observed below an altitude of $900 \mathrm{~km}$ on the dayside is associated with large fluxes of low-energy precipitating electrons and field-aligned currents. Block et al. (1987) reported a few cases of intense (up to $100 \mathrm{mv} / \mathrm{m}$ ) fluctuations around the dayside auroral oval and cleft at altitudes of 11000 to $13500 \mathrm{~km}$. Matsuoka et al. (1991, 1993) clarified the nature of low-frequency electric-field fluctuations in the frequency range of 0.5 to $3 \mathrm{~Hz}$ and their correlation with precipitating particles in the cusp. They identified the fluctuations as Alfvén waves propagating downward and suggested that the waves are generated in association with the injection of magnetosheath particles.

Published by Copernicus GmbH on behalf of the European Geosciences Union. 


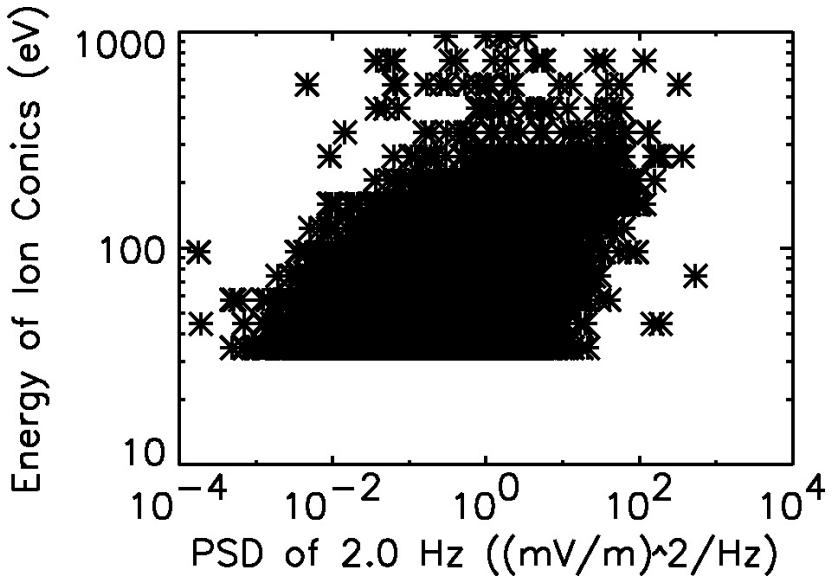

Fig. 1. Scatter plot of energy of ion conics and power spectral density of $2.0-\mathrm{Hz}$ component of LEFs.

Alfvén waves over the auroral region have been intensively studied in terms of charged particle acceleration. Smallscale Alfvén waves can carry a parallel electric field due to kinetic effects, causing field-aligned acceleration of electrons (Goertz and Boswell, 1979). Recent observations have confirmed the relationship between Alfvén waves and fieldaligned electron beams (e.g. Chaston et al, 2003; Tanaka et al., 2005).

Transverse ion heating (i.e. upwelling ions and /or ion conics) around the cusp/cleft region is possibly another consequence of these electric-field fluctuations (Gurnett et al., 1984; Lundin et al., 1990; Hultqvist, 1996; Stasiewicz et al., 2000).

Broad-band ELF waves have been found to correlate well with ion heating on the dayside and are most likely to be responsible for dayside ion heating (e.g. Andre and Yau, 1997; Norqvist et al., 1998), while Lundin et al. (1990) point out that the energy of transversely heated ions correlates well with the energy spectral density of low-frequency electricfield fluctuations. Hultqvist (1996) demonstrated that ions can be accelerated even up to several $\mathrm{keV}$ by the observed waveform of low-frequency electric-field fluctuations. It is possible that Alfvén waves may stimulate instabilities, resulting in waves being generated at frequencies approaching the ion cyclotron frequency (e.g. Wahlund et al., 1994; Seyler et al., 1998; Singh and Khazanov, 2004). An alternative explanation is the direct energization of ions by low-frequency Alfvén waves (e.g. Stasiewicz et al., 2000; Chaston et al., 2004).

Statistical studies of the evolution of ion conics versus altitude (Peterson et al., 1992; Miyake et al., 1993, 1996) show that the characteristic energy of ions increases with altitude, while their conic angle with respect to the upward direction decreases more slowly than would be expected from adiabatic folding, according to a height-integrated heating scenario. Transverse ion heating in the dayside cusp/cleft occurs in a region of extended longitude and altitude that is nevertheless well defined with a finite extent in latitude. This region is often called the polar-cusp heating wall (Knudsen et al., 1994). Ions enter the heating wall, are heated there for a time period, and then escape from the heating wall due to poleward convection. The effect of poleward convection along with the field-aligned motion of ions contributes to a specific dispersion pattern shown downstream of the heating wall. Recent observations and model calculations indicate that the heating wall is likely to extend up to an altitude of several Earth radii (Bouhram et al., 2004).

The purpose of this study is to present further evidence for LEFs being a probable energy source for transverse ion heating. We investigated the relationship between the energy of ion conics and the power spectral density of LEFs, and found that the relationship between ion conics and LEFs is well understood in terms of a scenario of height-integrated heating of ions in the heating wall.

\section{Data}

The data presented here were obtained by the polar-orbiting Akebono (Exos-D) satellite (Oya and Tsuruda, 1990; Tsuruda and Oya, 1991). The satellite was launched on 22 February 1989, into an elliptical orbit at an inclination of $75.1 \mathrm{deg}$, with an initial apogee of $10482 \mathrm{~km}$ and a perigee of $272 \mathrm{~km}$. The satellite is dedicated to the study of auroral particle acceleration and related physical phenomena over the polar region. It spins with a period of $8 \mathrm{~s}$ around an axis directed toward the Sun. An Electric-Field Detector (EFD) and Low-Energy Particle detector (LEP), along with six other scientific instruments, are installed on board the Akebono satellite. Observations have been successfully carried out since the instruments were first turned on and a large database has been built. The data used here were obtained from April 1989 through January 1992.

The EFD measures the electric field using two sets of double probes located at the tips of roughly 30-m wire booms which extend perpendicular to the spin axis (60-m tip to tip). The resolution is $0.06 \mathrm{mV} / \mathrm{m}$, but the accuracy is about $0.12 \mathrm{mV} / \mathrm{m}$, owing to instrumental noise. The sampling rate is $32 \mathrm{~Hz}$. We assume that there are no parallel electric fields along the magnetic field in deriving the three components of the electric field from two-dimensional measurements in the plane perpendicular to the spin axis. Even when there is a substantial parallel field accelerating the auroral particles, it is only a small fraction of the total electric field and does not significantly affect the derived electric field. Further information on the EFD instrument is available in Hayakawa et al. (1990). Miyake et al. (2003) surveyed the characteristics of occurrences of intense LEFs around the dayside cusp/cleft region. We use the same data sets for LEFs as in Miyake et al. (2003), which include the power spectral density of electric-field fluctuations between 0.2 and $4.0 \mathrm{~Hz}$. In this 
(a)

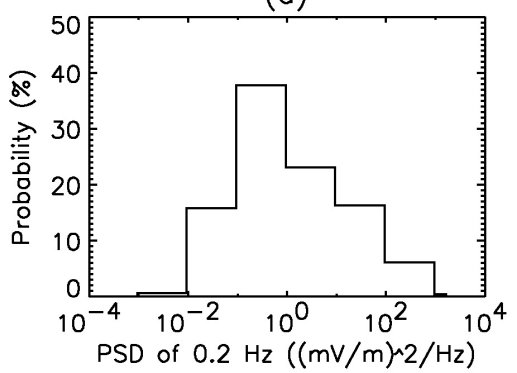

(c)

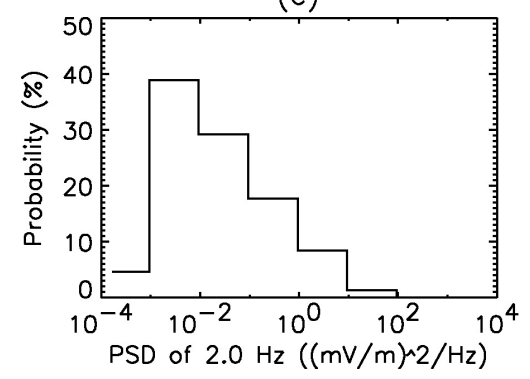

(b)

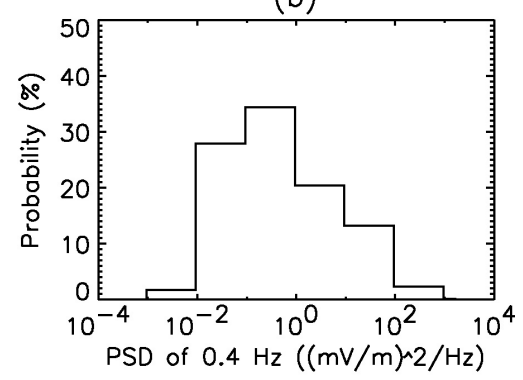

(d)

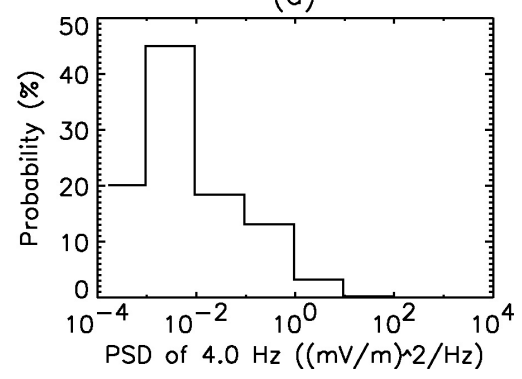

Fig. 2. Histograms of occurrence of power spectral density of LEFs at four frequencies. All data in our database (i.e. at an invariant latitude of 65-85 deg) are presented, including measurements over the polar cap and in the lower latitude region of the cusp/cleft and auroral oval.

paper, we use the term LEFs, rather than Alfvén waves, because we analyzed only electric-field data.

The particle data used in this study were obtained using an LEP instrument (Mukai et al., 1990) which consists of two sets of E/Q analyzers and was designed to observe energypitch angle distributions of auroral electrons and ions. It has an energy-per-charge range of $13 \mathrm{eV} / \mathrm{q}-20 \mathrm{KeV} / \mathrm{q}$ for ion measurement. The energy range is divided into 29 logarithmically spaced steps and is scanned in $2 \mathrm{~s}$. The pitch-angle range is divided into 18 bins of $10 \mathrm{deg}$ each in the dataprocessing procedure.

Observations have been successfully carried out since the instrument was initially turned on and a large data set for auroral electrons and ions has been built. Ion conics were automatically identified every $16 \mathrm{~s}$ by the algorithm used in Miyake et al. (1996). Details of the identification process and the statistical characteristics of dayside ion conics are also described by Miyake et al. (1996). We used the ions' energy and conic angle in the following analysis. The energy and conic angle are defined as the highest energy of conical distribution of ions in velocity space and as the angle of those most enegetic ions. We analyzed here the data of electric field and ions observed from 09:00 to 15:00 MLT and within an invariant latitude of $65-85 \mathrm{deg}$. The altitude range is from $5000 \mathrm{~km}$ to the satellite apogee.

\section{Correlation between LEFs and ion conics}

Figure 1 shows a scatter plot of the energy of ion conics and the power spectral density of the $2.0-\mathrm{Hz}$ component of
LEFs. A lower energy cutoff for ion conics is evident at $30 \mathrm{eV}$, which comes from the lower limit of our identification algorithm for ion conics. The energy of ion conics is positively correlated with the power spectral density of LEFs. The spectrum of LEFs generally has a power-law shape (see, for example, Kintner, 1976; Miyake et al., 2003) and similar correlations are found over the whole frequency range $(0.2-4.0 \mathrm{~Hz})$. This positive correlation was also reported in Lundin et al. (1990), who showed that the energy of ion conics has a high correlation with power spectral density not only at the local ion-cyclotron frequency, but also at any frequency of LEFs.

Figure 2 shows histograms of the occurrence of the power spectral density of LEFs at four frequencies. All the data in our data base (i.e. with an invariant latitude of $65-85 \mathrm{deg}$ ) are presented, including measurements made over the polar cap and in the lower latitude region of the cusp/cleft and auroral oval. The distribution is, therefore, somewhat concentrated at a low power spectral density for all the frequencies. A comparison with Fig. 1 shows that LEFs associated with ion conics have a high power spectral density.

Since the scatter plot in Fig. 1 is unsuitable for making a detailed comparison with Fig. 2, in Fig. 3 we present histograms of the power spectral density of LEFs associated with ion conics. The solid, dotted, and broken lines in each panel represent histograms of the power spectral density associated with ion conics within the energy ranges of 30-50, 50-100, and more than $100 \mathrm{eV}$, respectively. The average energy at each energy range is given at the bottom of the histogram. The occurrence of the power spectral density is 

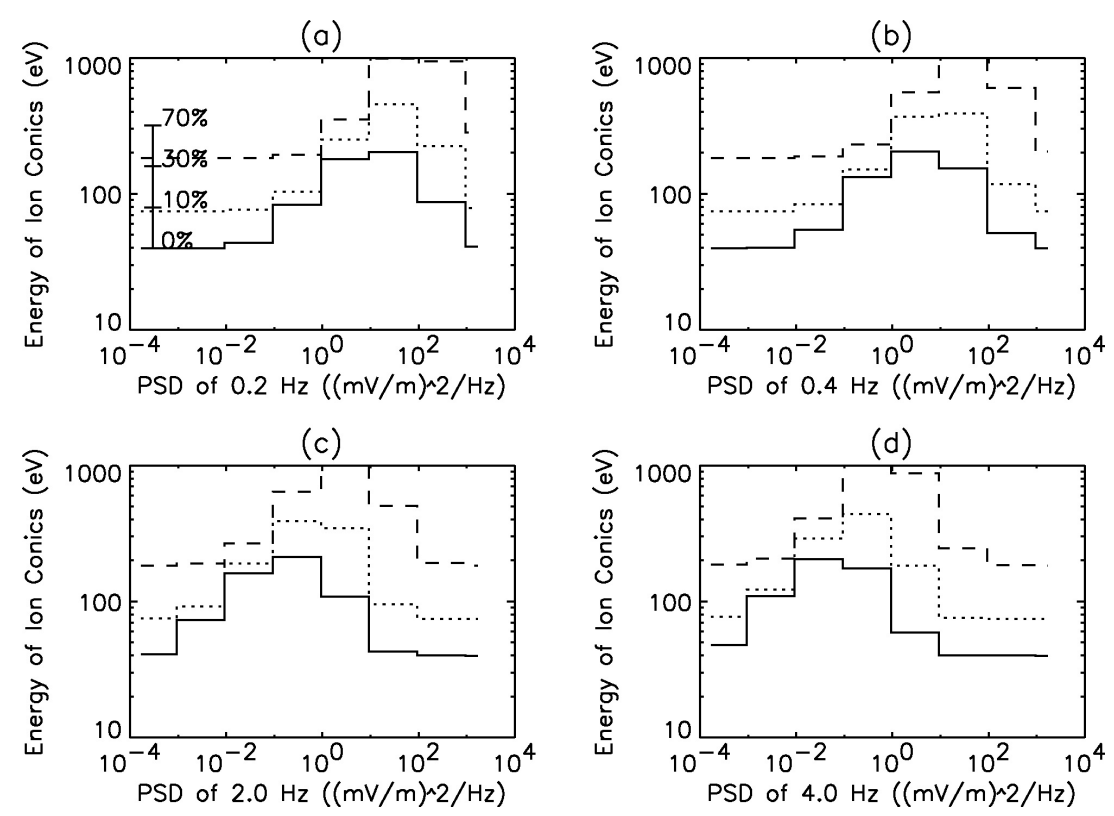

Fig. 3. Histograms of power spectral density of LEFs associated with ion conics. Solid, dotted, and broken lines represent histograms of power spectral density associated with ion conics within energy ranges of $30-50 \mathrm{eV}, 50-100 \mathrm{eV}$, and more than $100 \mathrm{eV}$, respectively. Occurrence is normalized to give a total of $100 \%$ in each histogram.
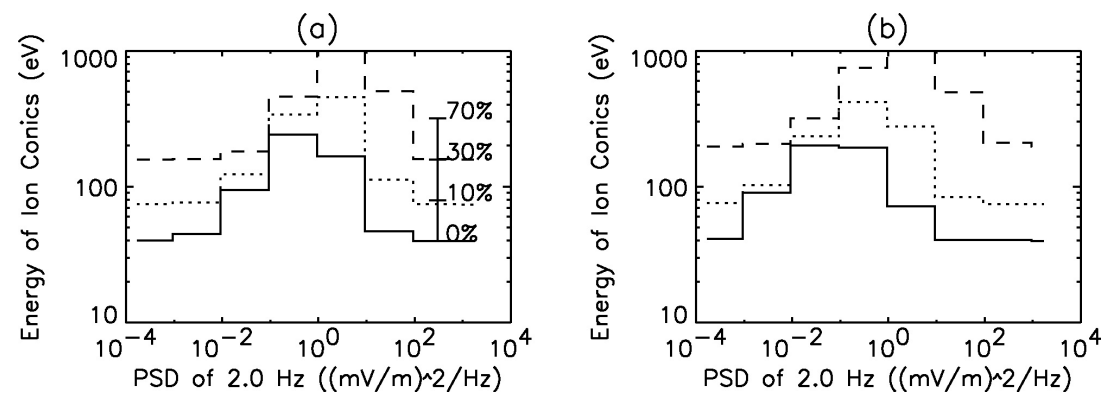

Fig. 4. Relationship of energy of perp. (a) and non-perp. (b) conics to power spectral density of LEFs. Format is same as that used in Fig. 3.

presented using the logarithmic scale given in Fig. 3a. Each histogram is normalized to give a total occurrence of $100 \%$.

The power spectral density of LEFs decreases with increasing frequency, reflecting the general characteristics of the power-law spectrum of LEFs. As Fig. 1 shows, there is a positive correlation of the energy of ion conics with the power spectral density of LEFs at all frequencies. Compared with Fig. 2, it is evident that the distribution of the power spectral density of LEFs associated with ion conics occupies the higher portion of the entire distribution of the power spectral density. The power spectral density of LEFs associated with high-energy ion conics is the highest among the entire distribution. We conclude that ion conics are associated with LEFs of high power spectral density and, in particular, that the ion conics with the highest energy are associated with
LEFs with the highest power spectral density. These results suggest a strong connection between transverse ion energization and LEFs.

Knudsen et al. (1994) presented a polar-cusp heating-wall model for ion energization. The heating wall extends not only along the field line but also longitudinally. The ions are convected poleward and enter the heating wall from its low-latitude boundary. They are energized quickly near the low-latitude boundary (Whalen et al., 1991). The energy of ion conics is given by integration of the heating rate over the orbit of the ions while they traverse the heating wall; the magnetic mirror force converts the perpendicular energy to parallel energy. Further poleward convection promotes the escape of the ions from the heating region, which is rather thin in the latitudinal direction. 

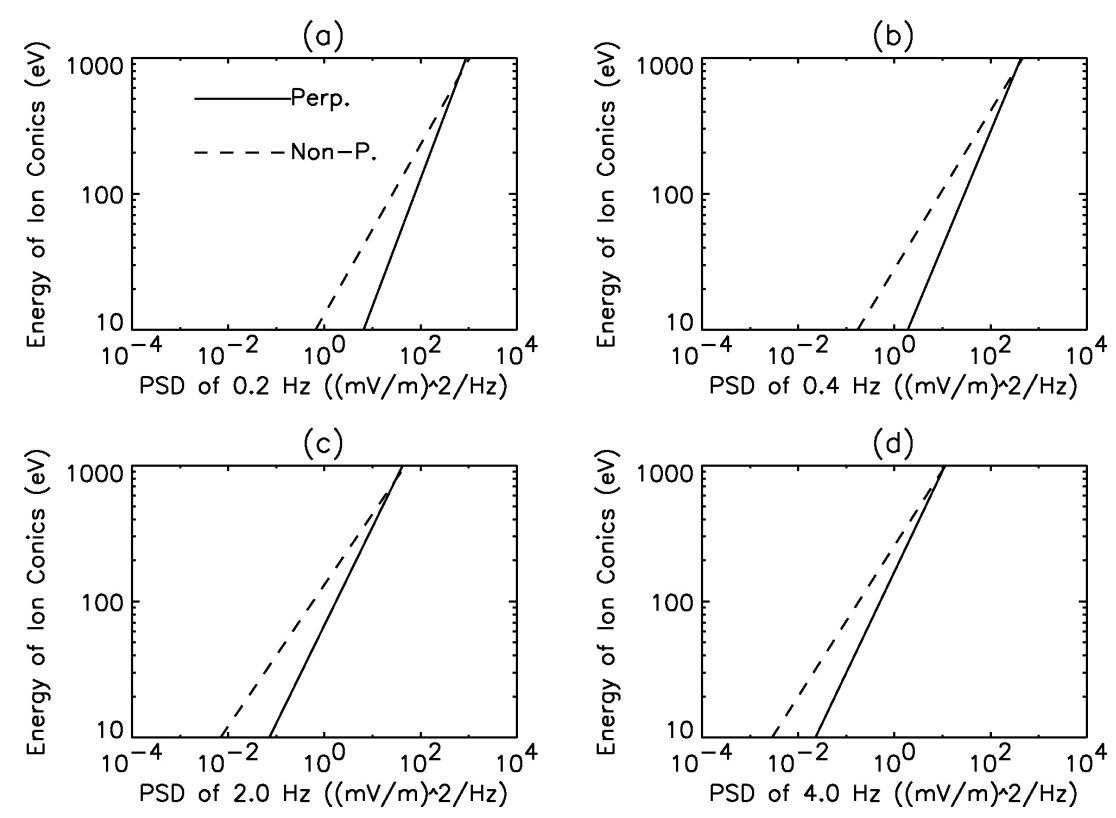

Fig. 5. Regression lines of energy of ion conics with power spectral density of LEFs. Solid and broken lines stand for relationship with perp. and non-perp. conics, respectively.
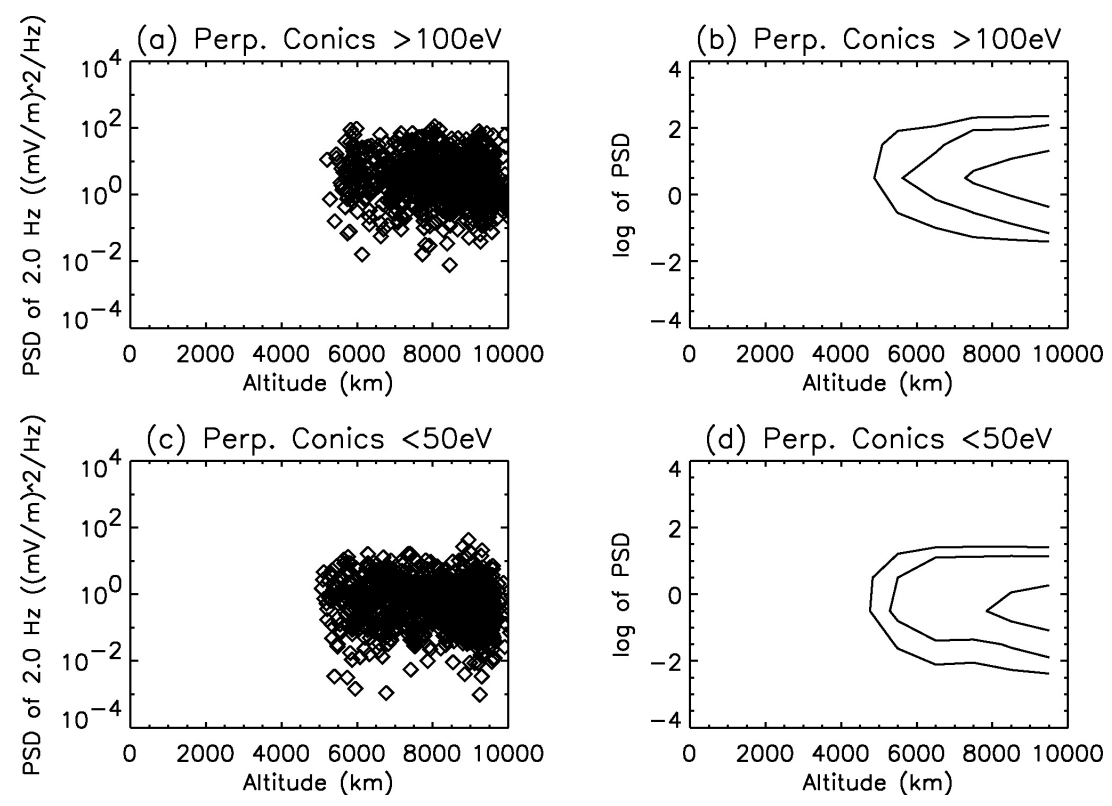

Fig. 6. Power spectral density at $2.0 \mathrm{~Hz}$ associated with perp. conics as a function of altitude. Upper and lower panels show those associated with high- and low-energy ion conics, respectively.

According to this scenario, ion conics with a conic angle perpendicular to the field line are located in the midst of the heating region, while ion conics folded to the field line tend to be much further from, at least, the most intense heating region. If LEFs are closely related to the transverse energization of ions, then the relationship should depend on the conic angle of the ions.
Figure 4 shows the relationship between the energy of near-perpendicular and folded ion conics and the power spectral density of LEFs. The format of the figure is the same as in Fig. 3. Figure 4a (left) shows ion conics with a conic angle of more than 60 deg and Fig. $4 \mathrm{~b}$ (right) shows those with an angle of less than $60 \mathrm{deg}$. 

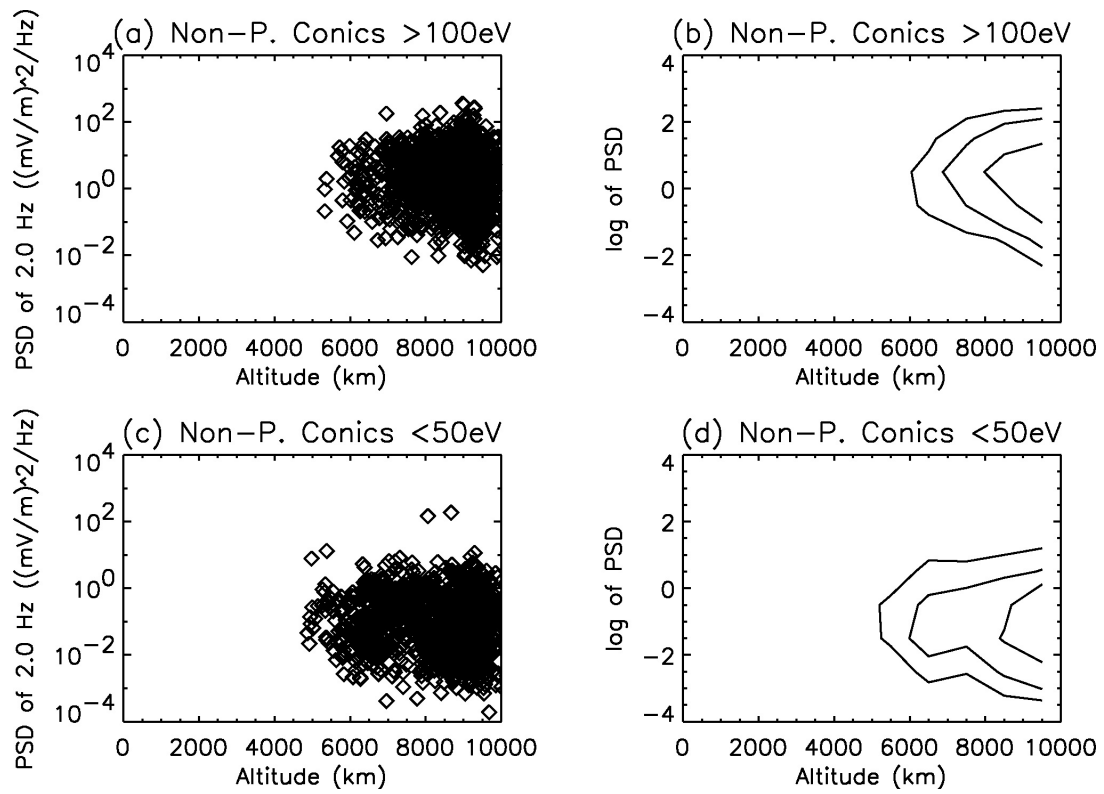

Fig. 7. Power spectral density at $2.0 \mathrm{~Hz}$ associated with non-perp. conics as a function of altitude. Upper and lower panels are those associated with high- and low-energy ion conics, respectively.

Hereafter, we call ion conics with a conic angle of more than $60 \mathrm{deg}$ "perp. conics" and those with an angle of less than $60 \mathrm{deg}$ "non-perp. conics". The energy of both types of ion conics is positively correlated with the power spectral density of LEFs, but the intensity of the power spectral density is higher for perp. conics. This tendency is more pronounced at a lower energy. Here, only the relationship with the power spectral density at $2.0 \mathrm{~Hz}$ is given, but the relationships are similar for other frequencies.

To differentiate between perp. and non-perp. conics more clearly, regression lines are plotted in Fig. 5. The solid and broken lines stand for the relationship with perp. and nonperp. conics, respectively. As Fig. 4 shows, perp. conics are generally associated with LEFs which have a higher power spectral density, and this tendency is clearer at a lower energy. The correlation coefficient in the logarithm was about 0.5 for both perp. and non-perp. conics at the four frequencies.

In addition to the intensity of the power spectral density being higher at a lower frequency, which comes from the nature of the power-law spectrum of LEFs, there is another remarkable dependency on frequency. The gradient of the regression line seems to be steeper at lower frequencies. In other words, LEFs associated with higher-energy conics not only tend to have higher intensity but also a harder spectral index. The spectral index $\gamma$ is defined by the inferred relation $\mathrm{E} \propto \mathrm{S}^{\gamma}$, where $\mathrm{E}$ and $\mathrm{S}$ are the energy and power spectral densities, respectively. The indexes of perp. and non-perp. conics are 0.91 and 0.62 at $0.2 \mathrm{~Hz}$, whereas at $4.0 \mathrm{~Hz}$ they are 0.71 and 0.56 .
We investigated the altitude dependence of the relationship between the energy of ion conics and the power spectral density of LEFs. The power spectral density at $2.0 \mathrm{~Hz}$ associated with perp. conics as a function of altitude is shown in the left panels ( $\mathrm{a}$ and $\mathrm{c}$ ) of Fig. 6. The right panels (b and d) of the figure show contour plots of the distribution in the left panels. The upper panels relate to high-energy ion conics and the lower ones to low-energy ion conics. Figure 7 shows the same information for non-perp. conics, using the same format as in Fig. 6.

The lower cutoff at $5000 \mathrm{~km}$ of altitude results from the altitude limit of our database and is not real. There seems to be a slight decrease in the intensity of LEFs with increasing altitude. The correlation coefficient between the intensity of LEFs and the energy of ion conics is almost equivalent for perp. and non- perp. conics (Fig. 4). If we take the correlation at a narrow altitude range, the correlation coefficient for perp. conics should be higher.

We should consider the definition of the "perp." conics, i.e. the "perp." conics contain all the conics with a conic angle larger than $60 \mathrm{deg}$. The occurrence of conics with a conic angle larger than $80 \mathrm{deg}$ has a peak around an altitude of $5000-6000 \mathrm{~km}$ and is decreased with altitude (Miyake et al., 2000a). Therefore, the intensity of LEFs around an altitude of $5000-6000 \mathrm{~km}$ is close to that associated with perpendicular conics, while the intensity of LEFs around 9000$10000 \mathrm{~km}$ is close to that associated with 60 -deg conics. The difference in the major conic angle for perp. conics is responsible for the slight dependence on altitude in Fig. 6.

More non-perp. conics are found at a higher altitude due to conic angle folding resulting from the magnetic mirror force 
(a)

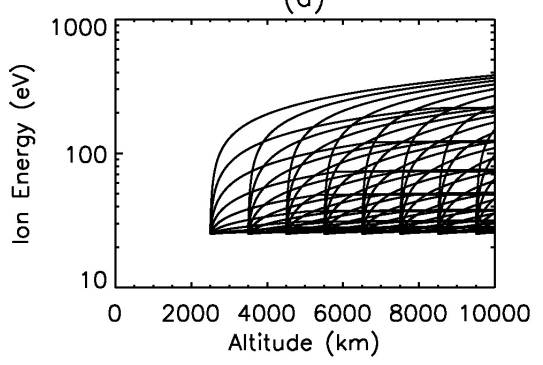

(c)

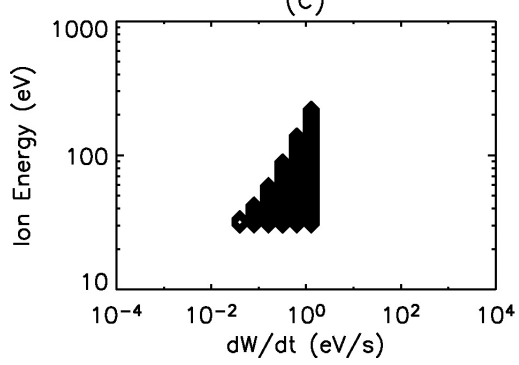

(b)

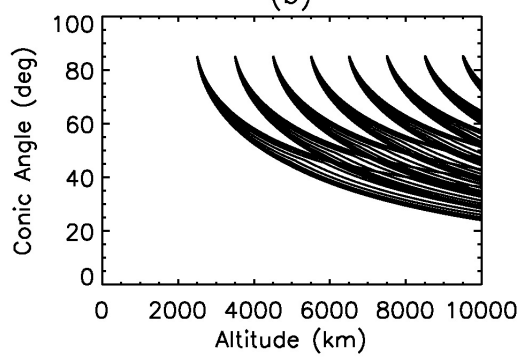

(d)

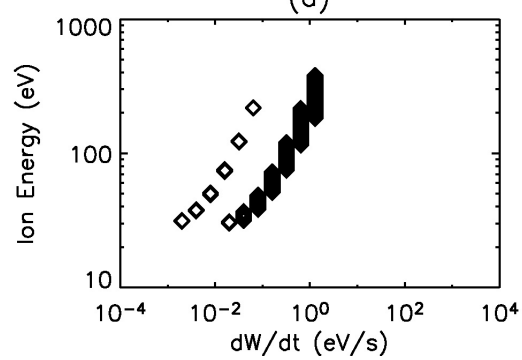

Fig. 8. Variations in energy (a) and conic angle (b) of each ion as a function of altitude and statistical summary of ion energy of perp. (c) and non-perp. (d) conics as a function of heating rate observed at altitudes between 5000 and $10000 \mathrm{~km}$.

(Fig. 7). The intensity of LEFs is more widespread for nonperp. conics, especially at high altitude, whereas it is more concentrated for perp. conics at a certain altitude in a specific energy range. It takes time for ion conics to fold to the field line, so it is possible that non-perp. conics sometimes experience some variations in the intensity of LEFs when they drift in the heating region. The local intensity of LEFs is not always the average intensity of the LEFs in which the ions are drifting, indicating that there are sometimes slight variations in the intensity of LEFs.

\section{Comparison with a simple model calculation}

To examine the idea that ions are transversely energized with a fraction of the energy of LEFs, we made a simple model of ion energization. The altitude along the field line was the only spatial dimension that we considered and we assumed oxygen ions. The ions had $25 \mathrm{eV}$ of energy and an initial conic angle of $85 \mathrm{deg}$. They were positioned at every $1000 \mathrm{~km}$ of altitude from 1500 through $9500 \mathrm{~km}$. We give various intensities for the wave field in calculating the model, but the intensity was assumed to be constant with altitude since the observation results show a significant correlation between the energy of non-perp. conics and the intensity of LEFs. If large variations in LEFs occurred frequently during the ion-energization process, poor correlation would be expected for non-perp. conics.

In the actual calculations, we simply made the temporal variation in ion energy, $\mathrm{dW} / \mathrm{dt}$, proportional to the intensity of the wave field. Ion-cyclotron resonance heating, for example, satisfies this condition. Chang et al. (1986) gave $\mathrm{dW} / \mathrm{dt}=\mathrm{S} l^{*} \mathrm{q}^{2} / 2 \mathrm{~m}$, where $S l$ is the spectral energy density of the electric left-hand circular polarized component around the ion gyrofrequency resonated with ions, $q$ is the charge of the ions, and $m$ is their mass. The energy conversion from $\mathrm{W}_{\perp}$ to $\mathrm{W}_{/ /}$in the terrestrial magnetic field is given by $\mathrm{dW}_{/ /} / \mathrm{dr}=3 \mathrm{~W}_{\perp} / \mathrm{r}$, where $r$ is the geocentric distance and $\mathrm{W}=\mathrm{W}_{\perp}+\mathrm{W}_{/ /}$.

The results of the model calculation are summarized in Figs. 8 and 9. Variations in the energy and conic angle of each ion are shown as a function of altitude in Figs. 8a and $b$. The ions were positioned at an altitude of every $1000 \mathrm{~km}$ from 1500 through $9500 \mathrm{~km}$ for the initial stage. Nine variations in the heating rate resulted in nine curves from the initial energy and conic angle at each launch altitude. The lower panels, Fig. $8 \mathrm{c}$ and d, show a statistical summary of the ion energy observed at altitudes between 5000 and $10000 \mathrm{~km}$, which is the same altitude range as the actual observations. For comparison with the observations, we selected ions with energies higher than $30 \mathrm{eV}$ and divided them into perp. (Fig. 8c) and non-perp. conics (Fig. 8d).

The ions first appear in Fig. 8c. The energy of the ions increased in a heating region at a constant rate. In contrast, the conic angle decreased, becoming less than $60 \mathrm{deg}$. The plot is then switched from Figs. 8c to d, showing that the ion energy of non-perp. conics is always higher than that of perp. conics at a certain heating rate. The energy at the switchover is higher at a higher heating rate, which leads to a positive correlation even for perp. conics (Fig. 8c).

In reality, ions never stay in a constant heating situation for long. For example, ions are convected poleward and finally 

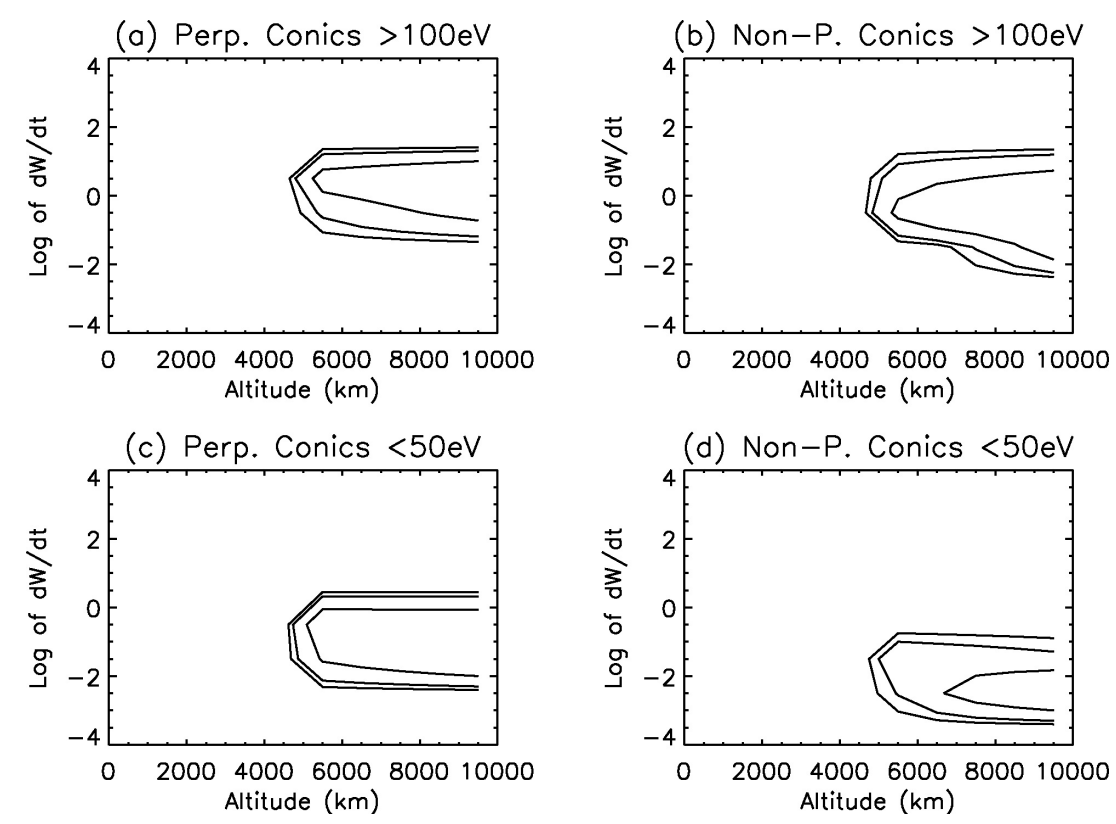

Fig. 9. Contour plots of altitude distribution of heating rate sorted by energy and conic angle (i.e. perp. and non-perp.) of ions, which represents the counterpart of the right panels of Figs. 6 and 7. Lower cutoff at $5000 \mathrm{~km}$ of altitude is imposed for consistency with actual observations and is not real.

even escape from the polar-cusp heating wall. The effect accounts for the wide spread intensity of LEFs associated with non-perp. conics at high altitude in Fig. 7. We took this effect into account in our model by abruptly decreasing the heating rate by an order of magnitude when $150 \mathrm{~s}$ had passed from the start of ion heating. This resulted in the second trend shown in Fig. 8d and also in a clearer differentiation between non-perp. and perp. conics, as shown in Fig. 8c. An abrupt variation after 150 s seems overly simple compared with the real situation. However, considering a latitude width of 12 deg for the heating wall (Knudsen et al., 1994; Dubouloz et al., 1998; Bouhram et al., 2004) and the convection velocity, this range of time is quite reasonable. A gradual change simply distributes the points between the original and second trends shown in Fig. 8d.

Figures $8 \mathrm{c}$ and $\mathrm{d}$ can be directly compared with Fig. 5 only if we assume that the heating rate is proportional to, or at least positively correlated with, the intensity of the LEFs. Although we took a single-particle approach with a great deal of simplification, and our purpose was simply to examine the basic idea, the two main features of the results of the observations were reproduced to a remarkable degree in the model calculation, that is, non-perp. conics generally had higher energy and there was a greater difference between perp. and non-perp. conics at a low energy.

Figure 9 shows contour plots of the altitude distribution of the heating rate sorted by the energy and conic angle (i.e. perp. and non-perp.) of the ions. These represent the counterpart of the right panels of Figs. 6 and 7. A lower cutoff at an altitude of $5000 \mathrm{~km}$ was imposed for consistency with the observations and does not reflect the actual situation.

Some of the basic features of the observation were reproduced in the model calculation. More non-perp. conics are found at higher altitudes due to conic angle folding caused by the magnetic mirror force. The intensity of LEFs has a wider spread for non-perp. conics at high altitude. This is due to the contribution of the second trend in Fig. 8d, i.e. the abrupt decrease in the intensity of LEFs when $150 \mathrm{~s}$ have passed from the start of ion heating. It takes time for ion conics to fold to the field line, so it is possible that non-perp. conics sometimes experience variations in the intensity of the LEFs while they drift in a heating region.

\section{Discussion}

Ion conics are associated with intense LEFs. The energy of ion conics is positively correlated with the power spectral density of LEFs. Lundin et al. (1990) reported a similar correlation from Viking observations. Both findings suggest a close relationship between LEFs and transverse ion energization.

The dependence of the correlation on the conic angle gives insights into this relationship and the process of ion energization. Although ion conics with a conic angle near $90 \mathrm{deg}$ (perp. conics) and those more aligned with magnetic field lines (non-perp. conics) both have an equivalent correlation coefficient with the local intensity of LEFs, the LEFs associated with near-perpendicular ion conics are generally more intense than those associated with folded conics. In other 
words, non-perp. conics have higher energy than perp. conics. This feature is more pronounced for lower energy ion conics.

This observation is consistent with a scenario of heightintegrated ion energization in the polar-cusp heating wall under poleward convection. The longer residence time for non-perp. conics leads to higher energy of the ions at a certain level of intensity of the LEFs. The longer period after entering the heating wall may tend to increase the possibility of ions escaping from the most intense heating region. Lower energy ions are obviously more susceptible to this effect. Since the $\boldsymbol{E} \times \boldsymbol{B}$ drift velocity is independent of ion energy and mass, the trajectory of high-energy ions is more field-aligned and that of low-energy ions is more horizontal. Therefore, high-energy ions tend to stay in the heating region in this altitude range, and the difference between perp. and non-perp. conics is clearer with lower energy. A simple model calculation supports this interpretation. Although we took a single-particle approach, the model reproduced the results of the observations very well.

We correlated the local intensity of the LEFs with the energy of local ion conics. Ions observed at a specific point were energized, not in the field of the LEFs at the observation point, but in the field of the LEFs below the observation point during their upward motion. Although this was more often the case for non-perp. conics, we still obtained a good correlation for non-perp. conics, which may be attributed to the stability of the LEFs in terms of both time and altitude. If ion heating was localized and there were large variations in altitude, non-perp. conics would never correlate well with the intensity of LEFs. The electric field of traveling Alfvén waves is obviously well correlated along the magnetic field line between different altitudes.

Miyake et al. (2003) surveyed some characteristics of the occurrence of intense LEFs around the cusp/cleft regions. Intense LEFs penetrate to lower altitudes, especially in the prenoon region. Ivchenko and Marklund (2001) also reported an intense, low-frequency electric-field fluctuation around the pre-noon region at an altitude of $1000 \mathrm{~km}$. Similarly, intense fluctuations in the magnetic field are found to penetrate low altitudes in the pre-noon region (Hasunuma et al., 2006 ${ }^{1}$ ). Ion conics also have an occurrence peak around the pre-noon region (e.g. Miyake et al., 1996). Intense LEFs are more frequently observed when the magnitude of the IMF $B_{y}$ component is large and there is a high-velocity solar wind. These features are quite similar to those of ion conics (Miyake et al., 2000b, 2002).

One of the features of ion heating around the cusp region is the response to the solar-wind pressure pulse. Moore

\footnotetext{
${ }^{1}$ Hasunuma, T., Fukunishi, H., Nagatsuma, T., Kataoka, R., Takahashi, Y., Matsuoka, A., and Kumamoto, A.: Polar distribution of small-scale field-aligned currents and their relationship to the large-scale field-aligned current system, J. Geophys. Res., submitted, 2006.
}

et al. (1999) and Fuselier et al. (2002) reported intensification of the outflow of ions in response to abrupt increases in the solar-wind dynamic pressure, which also suggests that a time-varying input of energy with a wide frequency range carried by Alfvén waves is responsible for ion heating around the cusp region.

This study focused on transverse ion energization around the dayside cusp/cleft region. The nightside polar-cap boundary is another region where intense LEFs and energetic ion outflow are observed (e.g. Nagatsuma et al., 1996; Miyake et al., 2001; Chaston et al., 2003). Recent FAST observations showed that auroral particle acceleration can be characterized by processes within upward and downward current regions, and polar-cap boundary acceleration regions (Paschmann et al., 2002). Alfvénic electric fields are dominant and dc field-aligned currents are variable and weak in the polar-cap boundary region. The intense ion heating transverse to magnetic field lines there may be interpreted according to the same processes as in the cusp/cleft region.

All of these findings indicate that part of the energy of LEFs is quite likely to be converted to transverse ion energy through some process. It is possible that Alfvén waves may stimulate instabilities that generate waves at frequencies approaching the ion-cyclotron frequency (e.g. Wahlund et al., 1994; Seyler et al., 1998; Singh and Khazanov, 2004). From Akebono observations, Kasahara et al. (2001) reported a positive correlation between ELF/VLF waves and transversely accelerated ions. An alternative explanation is direct energization of the ions by low-frequency Alfvén waves (e.g. Stasiewicz et al., 2000; Chaston et al., 2004). However, the purpose of this paper is to present further evidence to support the view that LEFs are the probable energy source for transverse ion energization, rather than to identify the mechanism that converts the energy of Alfvén waves to ions. In either mechanism, only a small fraction of the energy of LEFs is enough to account for ion energization, since LEFs have a large amount of energy (Lundin et al., 1990).

\section{Conclusions}

We found a good correlation between the energy of ion conics and the power spectral density of LEFs (Alfvén waves) over a wide frequency range. Near-perpendicular ion conics are associated with more intense LEFs, or equivalently, folded ion conics generally have higher energy than nearperpendicular ion conics. The difference is more pronounced in the lower energy range. The dependence of the relationship of ion energy versus LEF intensity on the conic angle is well explained by gradual, quasi-constant, height-integrated heating of ions in the polar-cusp heating wall, along with the escape of ions from intense heating regions through poleward convection. These results suggest that part of the energy of LEFs is responsible for the transverse heating of ions. 
Acknowledgements. We thank all the members of the Exos-D project team, especially K. Tsuruda and H. Oya, for their extensive support.

Topical Editor T. Pulkkinen thanks M. Bouhram for his help in evaluating this paper.

\section{References}

Andre, M. and Yau, A. W.: Theories and observations of ion energization and outflow in the high latitude magnetosphere, Space Sci. Rev., 80, 27-48, 1997.

Block, L. P., Falthammar, C.-G., Lindqvist, P.-A., Marklund, G., Mozer, F. S., Pedersen, A., Potemra, T. A., and Zanetti, L. J.: Electric field measurements on Viking: First results, Geophys. Res. Lett., 14, 435-438, 1987.

Bouhram, M., Klecker, B., Miyake, W., Rème, H., Sauvaud, J.-A., Malingre, M., Kistler, L., and Blagau, A.: On the altitude dependence of transversely heated O+ distributions in the cusp/cleft, Ann. Geophys., 22, 1787-1798, 2004.

Chang, T., Crew, G. B., Hershkowictz, N., Jasperse, J. R., Retterer, J. M., and Winningham, J. D.: Transverse acceleration of oxygen ions by electromagnetic ion cyclotron resonance with broadband left-hand-polarized waves, Geophys. Res. Lett., 13, 636639, 1986.

Chaston, C. C., Bonnell, J. W., Carlson, C. W., Ergun, R. E., McFadden, J. P., and Strangway, R. J.: Properties of small scale Alfvén waves and accelerated electrons from FAST, J. Geophys. Res., 108(A4), 8003, doi:10.1029/2002JA009420, 2003.

Chaston, C. C., Bonnell, J. W., Carlson, C. W., McFadden, J. P., Ergun, R. E., Strangway, R. J., and Lund, E. J.: Auroral ion acceleration in dispersive Alfvén waves, J. Geophys. Res., 109, A04205, doi:10.1029/2003JA010053, 2004.

Dubouloz, N., Delcourt, D., Malingre, M., Berthelier, J.-J., and Chugunin, D.: Remote analysis of cleft ion acceleration using thermal plasma measurements from Interball Auroral Probe, Geophys. Res. Lett., 25, 2925-2928, 1998.

Fuselier, S. A., Collin, H. L., Ghielmetti, A. G., et al.: Localized ion outflow in response to a solar wind pressure pulse, J. Geophys. Res., 107, doi:10.1029/2001JA000297, 2002.

Goertz, C. K. and Boswell, R. W.: Magnetosphere-ionosphere coupling, J. Geophys. Res., 84, 7239-7246, 1979.

Gorney, D. J., Clarke, A., Croley, D., Fennell, J. F., Luhmann, L., and Mizera, P.: The distribution of ion beams and conics below 8000 km, J. Geophys. Res., 86, 83-89, 1981.

Gurnett, D. A., Huff, R. L., Menietti, J. D., Burch, J. L., Winningham, J. D., and Shawhan, S. D.: Correlated low-frequency electric and magnetic noise along the auroral field lines, J. Geophys. Res., 89, 8971-8985, 1984.

Hayakawa, H., Okada, T., Ejiri, M., et al.: Electric field measurement on the Akebono (EXOS-D) satellite, J. Geomag. Geoelectr., 42, 371-384, 1990.

Hultqvist, B.: On the acceleration of positive ions by high-latitude, large amplitude electric field fluctuations, J. Geophys. Res., 101, 27 111-27 122, 1996.

Ivchenko, N. and Marklund, G.: Observation of low frequency electromagnetic activity at $1000 \mathrm{~km}$ altitude, Ann. Geophys., 19, 643-648, 2001.

Kasahara, Y., Hosoda, T., Mukai, T., Watanabe, S., Kimura, I., Kojima, H., and Niitsu, R.: ELF/VLF waves correlated with trans- versely accelerated ion in the auroral region observed by Akebono, J. Geophys. Res., 106, 21 123-21 136, 2001.

Kintner, P. M.: Observations of velocity shear driven plasma turbulence, J. Geophys. Res., 81, 5114-5122, 1976.

Knudsen, D. J., Whalen, B. A., Abe, T., and Yau, A. W.: Temporal evolution and spatial dispersion of ion conics: evidence for a polar heating wall, in: Solar System Plasmas in Space and Time, Geophys. Monogr., Vol. 84, edited by: Burch, J. L. and Waite Jr., J. H., AGU, Washington, D.C., 163-169, 1994.

Kondo, T., Whalen, B. A., Yau, A. W., and Peterson, W. K.: Statistical analysis of upflowing ion beams and conics distribution at DE-1 altitudes, J. Geophys. Res., 95, 12 091-12 102, 1990.

Lundin, R., Gustafsson, G., Eriksson, A. I., and Marklund, G.: On the importance of high-latitude low-frequency electric field fluctuations for the escape of ionospheric ions, J. Geophys. Res., 95, 5905-5919, 1990.

Marklund, G. T., Blomberg, L.G., Falthammar, C. G., Erlandson, R. E., and Potemra, T. A.: Signatures of the high-altitude polar cusp and dayside auroral regions as seen by the Viking electric field experiment, J. Geophys. Res., 95, 5767-5780, 1990.

Matsuoka, A., Mukai, T., Hayakawa, H., Kohno, Y.-I., Tsuruda, K., Nishida, A., Okada, T., Kaya, N., and Fukunishi, H.: ExosD observations of electric field fluctuations and charged particle precipitation in the polar cusp, Geophys. Res. Lett., 18, 305-308, 1991.

Matsuoka, A., Tsuruda, K., Hayakawa, H., Mukai, T., Nishida, A., Okada, T., Kaya, N., and Fukunishi, H.: Electric field fluctuations and charged particle Precipitation in the cusp, J. Geophys. Res., 98, 11 225-11 234, 1993.

Maynard, N. C., Heppner, J. P., and Egeland, A.: Intense, variable electric fields at ionospheric altitude regions as observed by DE2, Geophys. Res. Lett., 9, 981-984, 1982.

Miyake, W., Mukai, T., and Kaya, N.: On the evolution of ion conics along the field line from EXOS D observations, J. Geophys. Res., 98, 11 127-11 134, 1993.

Miyake, W., Mukai, T., and Kaya, N.: On the origins of the upward shift of elevated (bi-modal) ion conics in velocity space, J. Geophys. Res., 101, 26 961-26969, 1996.

Miyake, W., Mukai, T., and Kaya, N.: Modeling of occurrence frequencies of ion conics as a function of altitude and conic angle, Ann. Geophys., 18, 47-55, 2000a.

Miyake, W., Mukai, T., and Kaya, N.: Interplanetary magnetic field control of dayside ion conics, J. Geophys. Res., 105, 23339 23344,2000 b.

Miyake, W., Yoshioka, R., Matsuoka, A., Mukai, T., and Nagatsuma, T.: Low-frequency electric-field fluctuations and fieldaligned electron beams around the edge of an auroral acceleration region, Ann. Geophys., 19, 389-393, 2001.

Miyake, W., Mukai, T., and Kaya, N.: Relationship of upflowing ion beams and conics around the dayside cusp/cleft region to the interplanetary conditions, Ann. Geophys., 20, 471-476, 2002.

Miyake, W., Matsuoka, A., and Hirano, Y.: A statistical survey of low-frequency electric field fluctuations around the dayside cusp/cleft region, J. Geophys. Res., 108, 1008, doi:10.1029/2002JA009265, 2003.

Moore, T. E.: Ionospheric mass ejection in response to a CME, Geophys. Res. Lett., 26, 2339-2342, 1999.

Mukai, T., Kaya, N., Sagawa, E., et al.: Low energy charged particle observations in the 'auroral' magnetosphere: first results from 
Akebono (Exos-D) satellite, J. Geomag. Geoelectr., 42, 479-496, 1990.

Nagatsuma, T., Fukunishi, H., Hayakawa, H., Mukai, T., and Matsuoka, A.: Field-aligned currents associated with Alfvén waves in the poleward boundary region of the nightside auroral oval, J. Geophys. Res., 101, 21 715-21 730, 1996.

Norqvist, P., Andre, M., and Tyrland, M.: A statistical study of ion energization mechanisms in the auroral region, J. Geophys. Res., 103, 23 459-23 474, 1998.

Oya, H. and Tsuruda, K.: Introduction to the Akebono satellite observations, J. Geomag. Geoelectr., 42, 367-370, 1990.

Paschmann, G., Haaland, S., and Treumann, R.: Auroral plasma physics, Space Sci. Rev., 103, 1-475, 2002.

Peterson, W. K., Collin, H. L., Doherty, M. F., and Bjorklumd, C. $\mathrm{M}$ : $\mathrm{O}+$ and $\mathrm{He}+$ restricted and extended (bi-modal) ion conic distributions, Geophys. Res. Lett., 19, 1439-1442, 1992.

Seyler, C. E., Clark, A. E., Bonnell, J., and Wahlund, J. E.: Electrostatic broadband ELF wave emission by Alfvén wave breaking, J. Geophys. Res., 103, 7027-7042, 1998.
Singh, N. and Khzanov, G.: Numerical simulation of waves driven by plasma currents generated by low-frequency Alfvén waves in a multi-ion plasma, J. Geophys. Res., 109, A05210, doi:10.1029/2003JA010251, 2004.

Stasiewicz, K., Bellan, P., Chaston, C., et al.: Small scale Alfvénic structure in the aurora, Space Sci. Rev., 92, 423-533, 2000.

Tanaka, H., Saito, Y., Asamura, K., Ishii, S., and Mukai, T.: High time resolution measurement of multiple electron precipitations with energy-time dispersion in high-latitude part of the cusp region, J. Geophys. Res., 110, A07204, doi:10,1029/2004JA010664, 2005.

Tsuruda, K., and Oya, H.: Introduction to the Exos-D (Akebono) project, Geophys. Res. Lett., 18, 293-295, 1991.

Wahlund, J. E., Louarn, P., Chust, T., Feraudy, H. de, Roux, A., Holback, G., Dovner, P.-O., and Holmgren, G.: On ion acoustic turbulence and the nonlinear evolution of kinetic Alfvén waves in aurora, Geophys. Res. Lett., 21, 1831-1834, 1994.

Whalen, B. A., Watanabe, S., and Yau, A. W.: Observations in the transverse ion energization region, Geophys. Res. Lett., 18, 725$728,1991$. 